\title{
The Pennsylvania-Torun search for planets around evolved stars with HET
}

\author{
Andrzej Niedzielski ${ }^{1}$, Alex Wolszczan ${ }^{2,3}$, Grzegorz Nowak ${ }^{1}$, \\ Paweł Zieliński ${ }^{1}$, Monika Adamów ${ }^{1}$ and Sara Gettel ${ }^{2,3}$ \\ ${ }^{1}$ Toruń Centre for Astronomy, Nicolaus Copernicus University, \\ Gagarina 11, 87-100 Toruń, Poland \\ email: Andrzej.Niedzielski@astri.uni.torun.pl \\ ${ }^{2}$ Department for Astronomy and Astrophysics, Pennsylvania State University, \\ 525 Davey Laboratory, University Park, PA 16802 \\ ${ }^{3}$ Center for Exoplanets and Habitable Worlds, Pennsylvania State University, \\ 525 Davey Laboratory, University Park, PA 16802
}

\begin{abstract}
Searches for planets around giants represent an essential complement to 'traditional' surveys, because they furnish information about properties of planetary systems around stars that are the descendants of the A-F main sequence (MS) stars with masses as high as $\sim 5 M_{\odot}$. As the stars evolve off the MS, their effective temperatures and rotation rates decrease to the point that their radial velocity variations can be measured with a few $m s^{-1}$ precision. This offers an excellent opportunity to improve our understanding of the population of planets around stars that are significantly more massive than the Sun, without which it would be difficult to produce abroad, integrated picture of planet formation and evolution. Since 2001, about $30 \mathrm{such}$ objects have been identified, including our five published HET detections (Niedzielski et al. 2007; Niedzielski et al. 2009a; Niedzielski et al. 2009b). Our work has produced the tightest orbit of a planet orbiting a K-giant identified so far $(0.6 \mathrm{AU})$, and the first convincing evidence for a multiplanet system around such as star (Niedzielski et al. 2009a). Our most recent discoveries (Niedzielski et al. 2009b) have identified new multiplanet systems, including a very intriguing one of two brown dwarf-mass bodies orbiting a $2.8 M_{\odot}, \mathrm{K} 2$ giant. This particular detection challenges the standard interpretation of the so-called brown dwarf desert known to exist in the case of solar-mass stars. Along with discoveries supplied by other groups, our work has substantially added to the emerging evidence that stellar mass positively correlates with masses of substellar companions, all the way from red dwarfs to intermediate-mass stars. We present current status and forthcoming results from the Pennsylvania-Torun Search for Planets performed with the Hobby-Eberly Telescope (HET) since 2004.
\end{abstract}

Keywords. planetary systems, stars: low-mass, brown dwarfs

\section{Introduction}

High precision radial velocities determination for MS stars more massive than $\sim 1.3 M_{\odot}$ are not possible due to small number of spectral features present in the spectra of these stars and significant rotational broadening. In search for planetary mass companions to intermediate mass stars (IMS) one may try to use transits or direct imaging. One may also obtain precise RV for intermediate mass stars after they leave the MS and become subgiants or giants cool enough to develop spectra reach in narrow lines. Detailed analysis of the IMS stars with planets shows that relative efficiency of these four approaches differs significantly. In the case of stars more massive that $\sim 1.6 M_{\odot} \mathrm{RV}$ search for planets around giants is the most efficient way while for stars more massive than $\sim 2.2 M_{\odot}$ it is the only way. The Pennsylvania- Torun Search for Plantes (PTPS) is devoted to radial velocity 


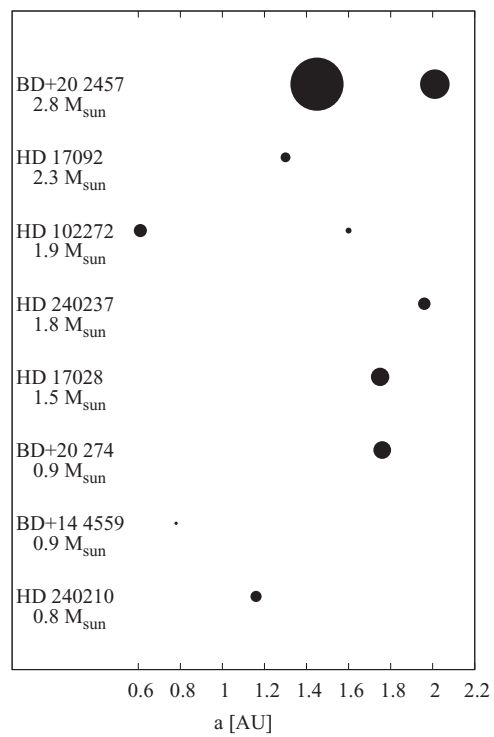

Figure 1. The first 10 substellar-mass companions discovered by PTPS. The symbol size is proportional to mass.

seach for and characterization of planets around stars more massive than the Sun with the Hobby - Eberly Telescope.

\section{Observations \& the sample}

Observations for the Pennsylvania - Torun Planet Search (PTPS) are obtained with the Hobby-Eberly Telescope (HET, Ramsey et al. 1998) equipped with the High Resolution Spectrograph (HRS, $\mathrm{R}=60.000$, Tull 1998) in the queue scheduled mode (Shetrone et al. 2007). The spectrograph was fed with a 2 arcsec fiber. The spectra consisted of 46 Echelle orders recorded on the 'blue' CCD chip (407-592 nm) and 24 orders on the 'red' one (602$784 \mathrm{~nm}$ ). Typical signal to noise ratio was 200-250 per resolution element. The basic data reduction was performed in a standard manner using IRAF tasks and scripts. RVs were measured using the standard $I_{2}$ cell calibration technique (Butler et al. 1996). Details of our survey, the observing procedure, and data analysis have been described in detail elsewhere (Niedzielski et al. 2007, Niedzielski \& Wolszczan 2008).

The sample of stars observed within PTPS is composed of about 350 giants from the Giant Clump, about 350 giants and subgiants and about 250 evolved MS stars on the upper envelope of the MS (aging dwarfs).

All stars are studied in detail to determine atmospheric parameters $\left(\mathrm{T}_{\text {eff }}, \operatorname{logg},[\mathrm{Fe} / \mathrm{H}]\right)$ and integrated parameters (mass, luminosity, age). Most of stars included in PTPS have masses ranging between 1 and 3 solar masses.

\section{Results}

For about 800 stars conclusive multi epoch observations were gathered. They allow us to subdivide our targets into single stars, stellar binaries and possible substellar-mass companions hosts. The three subsamples do not differ much in frequency of stellar binaries $\left(\sigma_{R V}>250 \mathrm{~ms}^{-1}\right)$ which ranges between $21 \%$ for the giants and subgiants sample and 
$27 \%$ for the aging dwarfs. The frequency of single stars $\left(\sigma_{R V}<20 \mathrm{~ms}^{-1}\right)$ varies from $45 \%$ in the clump giant sample to $62 \%$ for the giants and subgiants sample. Depending on criteria adopted the substellar-mass companion frequency may reach up to $27 \%$ in the case of the clump giant sample.

In Figure 1 the first 10 substellar-mass companions discovered by PTPS are presented. In addition to published already planets and brown dwarfs we present here also two more planets from Gettel et al. (2011) - BD+20 $274 \mathrm{~b}$ and HD $240237 \mathrm{~b}$ and from Nowak et al. (2011) - HD 17028 b.

\section{Acknowledgments}

We acknowledge the financial support from the Polish Ministry of Science and Higher Education through grants N203 510938 and N203 386237. AW acknowledges support from NASA grant NNX09AB36G. GN is a recipient of a graduate stipend of the Chairman of the Polish Academy of Sciences. We thank the HET resident astronomers and telescope operators for support. The Hobby-Eberly Telescope (HET) is a joint project of the University of Texas at Austin, the Pennsylvania State University, Stanford University, Ludwig-Maximilians-Universität München, and Georg-August-Universität Göttingen. The HET is named in honor of its principal benefactors, William P. Hobby and Robert E. Eberly. IRAF is distributed by the National Optical Astronomy Observatories and operated by the Association of Universities for Research in Astronomy, Inc., under cooperative agreement with the National Science Foundation.

\section{References}

Butler, R. P., Marcy, G. W., Williams, E., McCarthy, C., \& Dosanjh, P. 1996, PASP, 108, 500 Gettel, S. \& Wolszczan, A., et al. 2011, in preparation

Niedzielski, A., Konacki, M., Wolszczan, A., Nowak, G., Maciejewski, G., Gelino, C. R., Shao, M., Shetrone, M., \& Ramsey, L. W. 2007, ApJ, 669, 1354

Niedzielski, A. \& Wolszczan, A. 2008, Proc. IAU Symp., 249, 43

Niedzielski, A., Goździewski, K., Wolszczan, A., Konacki, M., Nowak, G., \& Zielinski, P. 2009a, ApJ, 693, 276

Niedzielski, A., Nowak, G., Adamów, M., \& Wolszczan, A. 2009b, ApJ, 707, 768

Nowak, G. \& Niedzielski, A. et al. 2011, in preparation

Ramsey, L. W. \& Adams, M. T. et al. 1998, Proc. SPIE, 3352, 34

Shetrone, M., Cornell, M., Fowler, J., et al. 2007, PASP, 119, 556

Tull, R. G. 1998, Proc. SPIE, 3355, 387 